\section{Normal-mode analysis of the structures of perovskites with tilted octahedra. Erratum}

\author{
C. N. W. Darlington
}

School of Physics and Astronomy, University of Birmingham, Birmingham B15 2TT, England. Correspondence e-mail: c.n.w.darlington@bham.ac.uk

There is an error in the mode assignment for hettotype 9, $\left[a^{-} b^{+} a^{-}\right]$, discussed in the paper by Darlington [Acta Cryst. (2002). A58, 66-71], which has been pointed out by Dr Kevin Knight, Rutherford Appleton Laboratory, Didcot, Oxon, England. In this paper, a mode involving displacements of the anions of hettotype 9 was labelled $\left[\left(\frac{1}{2}, 0, \frac{1}{2}\right), M_{1}\right]$ rather than $\left[\left(\frac{1}{2}, 0, \frac{1}{2}\right), M_{2}\right]$. Both modes involve plus-like distortion of the octahedra. In the corrected Tables 1-4 shown below, this mode, which is only found in hettotype 9 , has been labelled $K_{2}$ rather than $\mathrm{H}_{2}$. Therefore, there are not seven but eight normal modes of the cubic phase required to describe the displacements found in the nine hettotypes considered. The weights of $K_{2}$ in all the materials examined in the original paper with the structure of hettotype 9 [labelled $W\left(H_{1}\right)$ in the original Table 4] are correct, unaltered by the change in the labelling of the mode. It should be noted that $\left[\left(\frac{1}{2}, 0, \frac{1}{2}\right), M_{2}\right]$ is a longitudinal mode - the seven other modes are all transverse. The weights of $K_{2}$ are not significantly different from zero in the 15 structures examined.

\section{References}

Bochu, B., Deschizeaux, N. N., Joubert, J. C., Collomb, A., Chenevas, J. \& Marezio, M. (1979). J. Solid State Chem. 29, 291-298.

Chenevas, J., Joubert, J. C., Marezio, M. \& Bochu, B. (1975). J. Solid State Chem. 14, 25-32.

Darlington, C. N. W. \& Knight, K. S. (1999). Acta Cryst. B55, 24-30.

Howard, C. J. \& Kennedy, B. J. (1999). J. Phys. Condens. Matter, 11, 3229-3236.

Kennedy, B. J., Howard, C. J. \& Chakoumakos, B. C. (1999). Phys. Rev. B, 59, $4023-4027$.

Knight, K. S. (1995). Solid State Ionics, 75, 109-118.

Leinenweber, K. \& Parise, J. (1995). J. Solid State Chem. 114, 277-281.

Marezio, M., Remeika, J. P. \& Dernier, P. D. (1970). Acta Cryst. B26, 2008-2022.

Unoki, H. \& Sakudo, T. (1967). J. Phys. Soc. Jpn, 23, 546-552.

Wiseman, P. J. \& Dickens, P. (1976). J. Solid State Chem. 17, 91-100.
Table 1

The nine hettotypes.

\begin{tabular}{llllll}
\hline Number & $\begin{array}{l}+/- \\
\text { notation }\end{array}$ & {$\left[M_{i} R_{j}\right]$} & Multiplicity & True unit cell & Space group \\
\hline 1 & $a^{0} a^{0} c^{-}$ & $R_{3}$ & $2 \times 2 \times 2$ & $2^{1 / 2} \times 2^{1 / 2} \times 2$ & $I 4 / \mathrm{mcm}(140)$ \\
2 & $a^{-} b^{0} a^{-}$ & $R_{1}=R_{3}$ & $2 \times 2 \times 2$ & $2^{1 / 2} \times 2 \times 2^{1 / 2}$ & $\operatorname{Imma}(74)$ \\
3 & $a^{-} a^{-} a^{-}$ & $R_{1}=R_{2}=R_{3}$ & $2 \times 2 \times 2$ & $2^{1 / 2} \times 2^{1 / 2} \times 2^{1 / 2}$ & $R \overline{3} c(167)$ \\
4 & $a^{0} a^{0} c^{+}$ & $M_{3}$ & $2 \times 2 \times 1$ & $2^{1 / 2} \times 2^{1 / 2} \times 1$ & $P 4 / \mathrm{mbm}(127)$ \\
5 & $a^{+} a^{+} c^{0}$ & $M_{1}=M_{2}$ & $2 \times 2 \times 2$ & $2 \times 2 \times 2$ & $I 4 / \mathrm{mmm}(139)$ \\
6 & $a^{+} a^{+} a^{+}$ & $M_{1}=M_{2}=M_{3}$ & $2 \times 2 \times 2$ & $2 \times 2 \times 2$ & $\operatorname{Im} \overline{3}(204)$ \\
7 & $a^{0} b^{-} c^{+}$ & $R_{2}, M_{3}$ & $2 \times 2 \times 2$ & $2 \times 2 \times 2$ & $\mathrm{Cmcm}(63)$ \\
8 & $a^{+} a^{+} c^{-}$ & $M_{1}=M_{2}, R_{3}$ & $2 \times 2 \times 2$ & $2 \times 2 \times 2$ & $P 4_{2} / \mathrm{nmc}(137)$ \\
9 & $a^{-} b^{+} a^{-}$ & $R_{1}=R_{3}, M_{2}$ & $2 \times 2 \times 2$ & $2^{1 / 2} \times 2 \times 2^{1 / 2}$ & Pnma $(62)$ \\
\hline
\end{tabular}

Table 2

Atomic displacements in the seven normal modes, the symbol used in the construction of the Landau potential, and character of each mode.

\begin{tabular}{|c|c|c|c|}
\hline Normal mode & Displacement & Symbol & Character \\
\hline$\left(\frac{1}{2}, \frac{1}{2}, \frac{1}{2}\right), \Gamma_{25}$ & $\mathrm{OI}(y)=-\mathrm{OII}(z)$ & $R_{1}$ & Octahedral minus tilt \\
\hline$\left(\frac{1}{2}, \frac{1}{2}, \frac{1}{2}\right), \Gamma_{25}$ & $\mathrm{OI}(x)=-\mathrm{OIII}(z)$ & $R_{2}$ & Octahedral minus tilt \\
\hline$\left(\frac{1}{2}, \frac{1}{2}, \frac{1}{2}\right), \Gamma_{25}$ & $\mathrm{OII}(x)=-\mathrm{OIII}(y)$ & $R_{3}$ & Octahedral minus tilt \\
\hline$\left(\frac{1}{2}, \frac{1}{2}, \frac{1}{2}\right), \Gamma_{15}$ & $\mathrm{OI}(y)=\mathrm{OII}(z)$ & $G_{O 1}$ & Octahedral minus distortion \\
\hline$\left(\frac{1}{2}, \frac{1}{2}, \frac{1}{2}\right), \Gamma_{15}$ & $\mathrm{OI}(x)=\operatorname{OIII}(z)$ & $G_{O 2}$ & Octahedral minus distortion \\
\hline$\left(\frac{1}{2}, \frac{1}{2}, \frac{1}{2}\right), \Gamma_{15}$ & $\operatorname{OII}(x)=\operatorname{OIII}(y)$ & $G_{O 3}$ & Octahedral minus distortion \\
\hline$\left(\frac{1}{2}, \frac{1}{2}, \frac{1}{2}\right), \Gamma_{15}$ & $A(x)$ & $G_{A 1}$ & $A$ cation displacement \\
\hline$\left(\frac{1}{2}, \frac{1}{2}, \frac{1}{2}\right), \Gamma_{15}$ & $A(y)$ & $G_{A 2}$ & $A$ cation displacement \\
\hline$\left(\frac{1}{2}, \frac{1}{2}, \frac{1}{2}\right), \Gamma_{15}$ & $A(z)$ & $G_{A 3}$ & $A$ cation displacement \\
\hline$\left(0, \frac{1}{2}, \frac{1}{2}\right), M_{3}$ & $\mathrm{OI}(y)=-\mathrm{OII}(z)$ & $M_{1}$ & Octahedral plus tilt \\
\hline$\left(\frac{1}{2}, 0, \frac{1}{2}\right), M_{3}$ & $\mathrm{OI}(x)=-\mathrm{OIII}(\mathrm{z})$ & $M_{2}$ & Octahedral plus tilt \\
\hline$\left(\frac{1}{2}, \frac{1}{2}, 0\right), M_{3}$ & $\mathrm{OII}(x)=-\mathrm{OIII}(y)$ & $M_{3}$ & Octahedral plus tilt \\
\hline$\left(0, \frac{1}{2}, \frac{1}{2}\right), M_{1}$ & $\mathrm{OI}(y)=\mathrm{OII}(z)$ & $H_{1}$ & Octahedral plus distortion \\
\hline$\left(\frac{1}{2}, 0, \frac{1}{2}\right), M_{1}$ & $\mathrm{OI}(x)=\mathrm{OIII}(z)$ & $\mathrm{H}_{2}$ & Octahedral plus distortion \\
\hline$\left(\frac{1}{2}, \frac{1}{2}, 0\right), M_{1}$ & $\operatorname{OII}(x)=\operatorname{OIII}(y)$ & $\mathrm{H}_{3}$ & Octahedral plus distortion \\
\hline$\left(0, \frac{1}{2}, \frac{1}{2}\right), M_{2}$ & $\mathrm{OII}(y)=-\mathrm{OI}(z)$ & $K_{1}$ & Octahedral plus distortion \\
\hline$\left(\frac{1}{2}, 0, \frac{1}{2}\right), M_{2}$ & $\mathrm{OI}(z)=-\mathrm{OIII}(x)$ & $K_{2}$ & Octahedral plus distortion \\
\hline$\left(\frac{1}{2}, \frac{1}{2}, 0\right), M_{2}$ & $\operatorname{OIII}(x)=-\operatorname{OII}(y)$ & $K_{3}$ & Octahedral plus distortion \\
\hline$\left(\frac{1}{2}, 0,0\right), M_{5}^{\prime}$ & $\operatorname{OIII}(y)$ & $X_{O 12}$ & Octahedral distortion \\
\hline$\left(\frac{1}{2}, 0,0\right), M_{5}^{\prime}$ & $\operatorname{OIII}(z)$ & $X_{O 13}$ & Octahedral distortion \\
\hline$\left(0, \frac{1}{2}, 0\right), M_{5}^{\prime}$ & $\mathrm{OII}(z)$ & $X_{O 23}$ & Octahedral distortion \\
\hline$\left(0, \frac{1}{2}, 0\right), M_{5}^{\prime}$ & $\mathrm{OII}(x)$ & $X_{O 21}$ & Octahedral distortion \\
\hline$\left(0,0, \frac{1}{2}\right), M_{5}^{\prime}$ & $\mathrm{OI}(x)$ & $X_{O 31}$ & Octahedral distortion \\
\hline$\left(0,0, \frac{1}{2}\right), M_{5}^{\prime}$ & $\mathrm{OI}(y)$ & $X_{O 32}$ & Octahedral distortion \\
\hline$\left(\frac{1}{2}, 0,0\right), M_{5}^{\prime}$ & $A(y)$ & $X_{A 12}$ & Cation displacement \\
\hline$\left(\frac{1}{2}, 0,0\right), M_{5}^{\prime}$ & $A(z)$ & $X_{A 13}$ & Cation displacement \\
\hline$\left(0, \frac{1}{2}, 0\right), M_{5}^{\prime}$ & $A(z)$ & $X_{A 23}$ & Cation displacement \\
\hline$\left(0, \frac{1}{2}, 0\right), M_{5}^{\prime}$ & $A(x)$ & $X_{A 21}$ & Cation displacement \\
\hline$\left(0,0, \frac{1}{2}\right), M_{5}^{\prime}$ & $A(x)$ & $X_{A 31}$ & Cation displacement \\
\hline$\left(0,0, \frac{1}{2}\right), M_{5}^{\prime}$ & $A(y)$ & $X_{A 32}$ & Cation displacement \\
\hline
\end{tabular}


Table 3

The space group, possible condensed modes and non-zero pseudocubic spontaneous macrostrain in the nine hettotypes.

\begin{tabular}{|c|c|c|c|}
\hline Number & Space group & Allowed modes & Macrostrain \\
\hline 1 & I4/mcm (140) & $R_{3}$ & $\varepsilon_{11}=\varepsilon_{22} ; \varepsilon_{33}$ \\
\hline 2 & Imma (74) & $\begin{array}{l}R_{1}=R_{3} \\
G_{O 1}=\mathrm{G}_{O 3} \\
G_{A 1}=\mathrm{G}_{A 3}\end{array}$ & $\varepsilon_{11}=\varepsilon_{33} ; \varepsilon_{22} ; \varepsilon_{31}$ \\
\hline 3 & $R \overline{3} c(167)$ & $R_{1}=R_{2}=R_{3}$ & $\varepsilon_{11}=\varepsilon_{22}=\varepsilon_{33} ; \varepsilon_{23}=\varepsilon_{31}=\varepsilon_{12}$ \\
\hline 4 & $P 4 / m b m(127)$ & $M_{3}$ & $\varepsilon_{11}=\varepsilon_{22} ; \varepsilon_{33}$ \\
\hline 5 & I4/mmm (139) & $\begin{array}{l}M_{1}=M_{2} \\
H_{1}=H_{2} \neq H_{3}\end{array}$ & $\varepsilon_{11}=\varepsilon_{22} ; \varepsilon_{33}$ \\
\hline 6 & $\operatorname{Im} \overline{3}(204)$ & $\begin{array}{l}M_{1}=M_{2}=M_{3} \\
H_{1}=H_{2}=H_{3}\end{array}$ & $\varepsilon_{11}=\varepsilon_{22}=\varepsilon_{33}$ \\
\hline 7 & Cmcm (63) & $\begin{array}{l}R_{2} \\
M_{3} \\
G_{O 2} \\
G_{A 2} \\
H_{3} \\
X_{O 32} \\
X_{A 32}\end{array}$ & $\varepsilon_{11} ; \varepsilon_{22} ; \varepsilon_{33}$ \\
\hline 8 & $P 4_{2} / n m c(137)$ & $\begin{array}{l}R_{3} \\
M_{1}=M_{2} \\
G_{O 3} \\
H_{1}=H_{2} \\
X_{\mathrm{O} 13}=X_{O 23} \\
X_{A 13}=X_{A 23}\end{array}$ & $\varepsilon_{11}=\varepsilon_{22} ; \varepsilon_{33}$ \\
\hline 9 & Pnma (62) & $\begin{array}{l}R_{1}=R_{3} \\
M_{2} \\
G_{O 1}=G_{O 3} \\
G_{A 1}=G_{A 3} \\
K_{2} \\
X_{O 21}=X_{O 23} \\
X_{A 21}=X_{A 23}\end{array}$ & $\varepsilon_{11}=\varepsilon_{33} ; \varepsilon_{22} ; \varepsilon_{31}$ \\
\hline
\end{tabular}

Table 4

Weights of condensed modes in units of $\mathrm{u} \AA^{2}$.

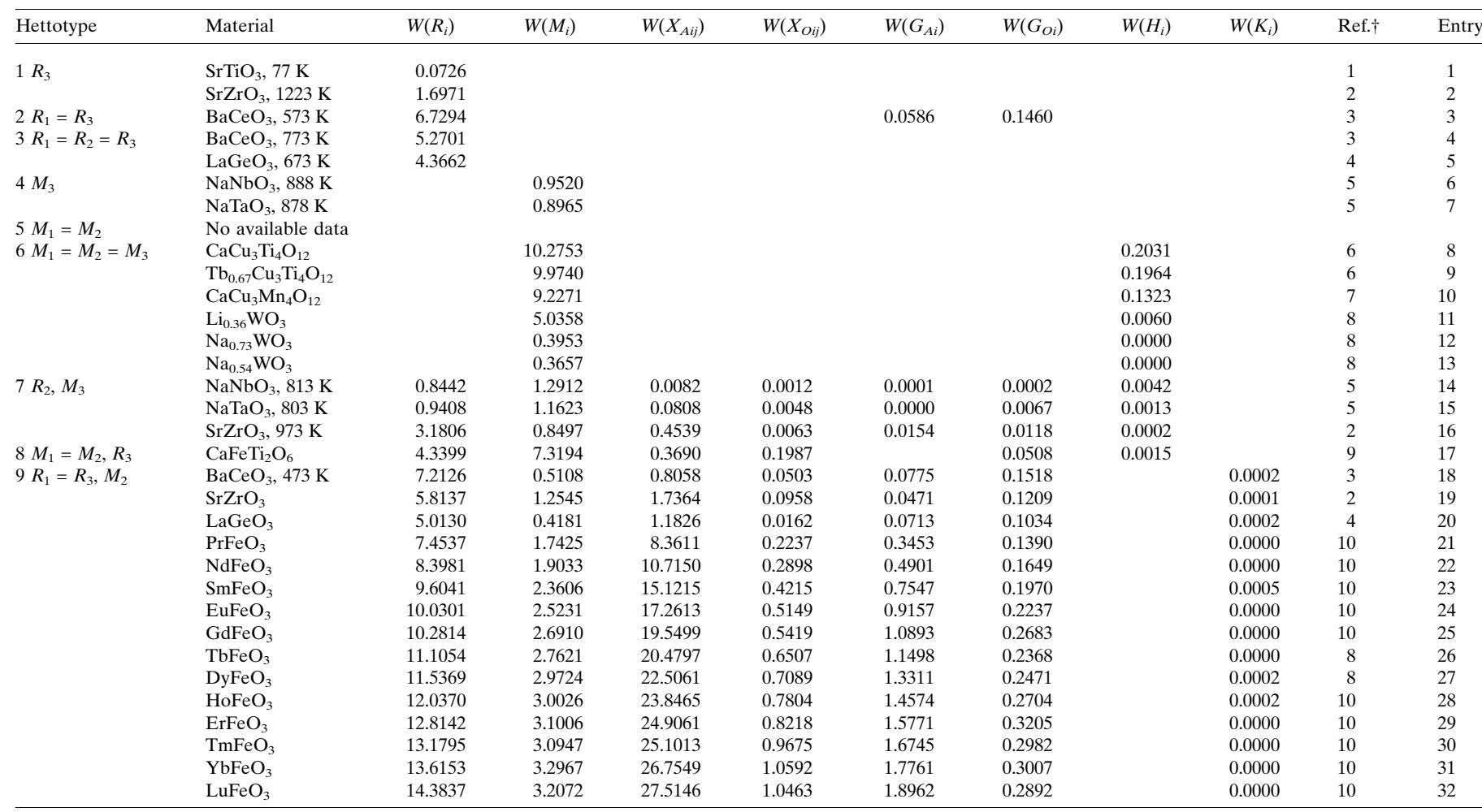

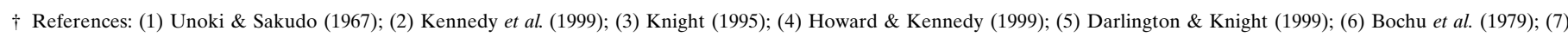
Chenevas et al. (1975); (8) Wiseman \& Dickens (1976); (9) Leinenweber \& Parise (1995); (10) Marezio et al. (1970). 